\title{
Methodology towards defining multi-scalar attributes of sustainability indicators in historical urban areas: assessment tool development
}

\author{
A. Nayer ${ }^{1,2} \&$ D. Abdel Fattah ${ }^{1,3}$ \\ ${ }^{1}$ College of Architecture and Design, Effat University, Saudi Arabia \\ ${ }^{2}$ Ministry of Higher Education, BHI High Institute of Technology, Egypt \\ ${ }^{3}$ Cairo University, Egypt
}

\begin{abstract}
Historical area conservation has become a cultural necessity not only for its urban qualities and valuable heritage but also due to the lack of maintenance and misuse of urban means. The need to protect the historical environment affected by deterioration of ecological resources and economic growth, in addition to high rates of consumption, is currently highly questionable. A framework for structuring information on sustainable urban indicators is discussed in order to develop a unique integrated representation that links together historical urban areas, socio-economic aspects, environmental, and spatial dimensions in addition to other factors such as time, space, activities, interests, and quality of life. In order to reduce the complexity of multi-attribute criteria of sustainability, this research proposes a model to assess urban sustainability indicators in heritage nodes. The research investigates indicators of urban qualities based on the integration of a BEQUEST network and efficient qualitative scales (quantifiable cities) to develop an integrative, multi-scalar assessment method. Delivered scheme, sustainable historical area index (SHAI), results should provide assessment methods to be used in developing historical areas and provides sustainable evaluations for enhancing urban aspects. A methodology towards maintaining the quality of urban approaches is considered as a means to evaluating the sustainable development of historical areas. This paper investigates the optimum corresponding values to be applied in assessing sustainability of urban development within historical areas.
\end{abstract}


Keywords: sustainability indicators, assessment methodology, historical urban areas, quality of urban spaces, multi-scalar attributes.

\section{Introduction}

There are broad concepts of sustainable development and varied sets of quantifiable criteria, or systems of indicators developed internationally and by local authorities in terms of sustainability processes within specific communities. However, it appears to be a necessity to customize such indicator systems relevant to urban historical areas. Therefore, measured aspects will be used to help planners, urban designers and other urban policy-makers with development issues $[1,2]$. Questions about the use of assessment methods to evaluate the sustainability of urban development (SUD) [3] have become key investigation topics in terms of case study applications. The framework and protocols BEQUEST [2] is devised into major aspects (as presented in figure 1).

Other sets of sustainability indicators have been selected relevant to the quantifiable city approach polygon: environment, economics, social, urban, and ecological. The five sided poles represent a link with a pre-established, international system of indicators that are related to the expected optimized urban fabric [1].

\section{Human economy}

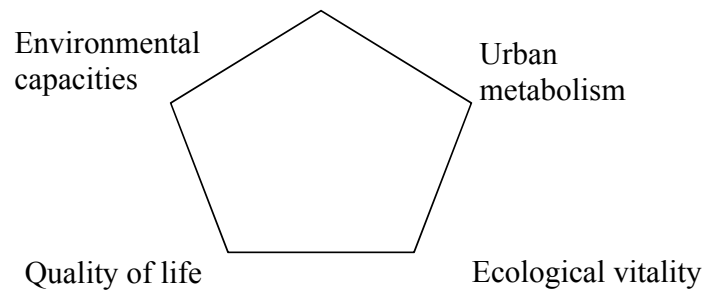

Figure 1: Quantifiable city.

Therefore, it is important to develop a concerted approach apprising the pooling of knowledge and understanding which best enhances sustainability within historical area development. It should be noted that establishing a consensus view across all members of the research team in this area will prove to be efficient and will further be sustained with investigated case studies in progressing stages [4]. Initially, a selection in respect of a wider range of disciplines and cultural contexts is under investigation in order to create principles with clear and distinct strategic meaning for the sustainable historical area index (SHAI).

\section{Sustainability aspects within urban historical areas}

Sustainable development requires a balanced coordination between different aspects, related to both the local social community and the historical urban 
setting. This will contribute to the concept of sustainable development; sustainable urban development is a prospect that meets the needs of present generations without compromising the ability of future generations to attain their imminent aspirations [3]. This remains the 'benchmark' definition around the concept and the conflict between the demand for human development and protection of historical urban fabrics into the future. The concept was further expanded at the Earth Summit, Rio 1992 (UNCED, 1992) in the Agenda 21 'Policy plan for environment and sustainable development in the 21 st Century'. These concepts are based upon the context of urban re/development $[2,3]$.

\subsection{Physical and non-physical qualities for sustainable urban development in historical areas}

The theory of the quantifiable city, basically defines sustainable urban development in terms of the five variables mentioned in figure 2 [1]. These variables are based on the urban sustainability domains (urban, social, economic, environmental, and ecological) which merge both physical and non-physical urban qualities [5].

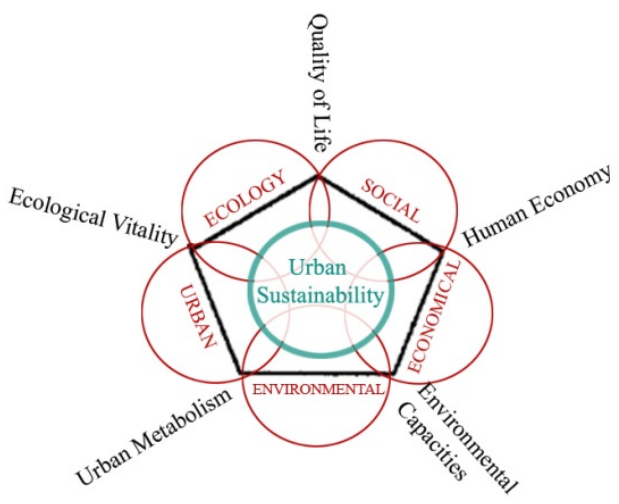

Figure 2: Quantifiable city.

Sustainable Urban Quality is a term with a wide range of approaches and definitions [6]. It can be considered as the identity of the local community, or it can be measured as the quality of life. The objective is to identify the physical and non-physical qualities for sustainable urban development in historical areas by implementing the approach of a multi-scalar attribute determining the urban sustainability indicators for historical conserved urban areas [2, 3].

These multi-scalar attributes that form the core of the SHAI model primarily depend on two main approaches:

First, the BEQUEST framework (evaluations of sustainable urban development indicators model): BEQUEST network is undertaken to develop an integrative assessment methodology setting out the types of assessment methods used in evaluation of sustainable urban historical areas) [5]. 
Second, the theory of the quantifiable city (this method assesses the environmental capacity - ecological integrity and equity of urban development plans, programs and projects, captured in terms of the built stock, transport, safety, security, health and wellbeing that constitutes a city's quality of life) $[2,3]$.

\subsection{Quantifiable city}

The analysis of the quantifiable city represented by the previously cited five main domains: urban, social, environmental, ecological and economical, the overlap between these five domains are a group of terms and terminologies, defining the relation and interaction between the social and economic aspects in an urban local community, through the human economy, which is the ability and affordability of a local community to be a part of the financial process as well as co-funding sustainable urban development in the historical areas [6].

The merge between the ecological and social domain offers the variable addressing the quality of life, giving value and standards of the living environment. Urban metabolism reflects the deep relation between the urban physical features of street width, building heights, and its relation with the environment, in terms of shads and shadow, air circulation, and landscaping. Analyzing the impact of the environment and studying the interactions among a local community can be clear in the ecological vitality [6]. Environmental capacities for urban sustainability emerged as a central theme in the Earth Summit's Agenda 21 in 1992, reiterated at the World Summit on Sustainable Development in 2002. It is a process that involves shifting societal attitudes, norms, and behaviors to pursue development that is environmentally sound with enduring outcomes, modifying and improving the mandates, roles, and functions of organizations, and strengthening individuals' capabilities [6].

In addition to all the parameters already discussed, the significance of the cultural and spiritual background in terms of the built historical areas and overall wellbeing of citizens must be taken into consideration [6, 7].

These domains and their corresponding variables are imperative towards quantifying all the dimensions associated with the quality of life and sustainable urban communities in historical areas [7].

On another level, the built urban environment (formed by physical and tangible variables) and its cultural historical heritage (intangible non-physical variables) is clearly important, while considered as a 'value and asset' that can be used to evaluate historical urban settings in terms of sustainability [7].

Thus, the quality of the urban environment in historical settings has become a key factor in the inter-competitiveness of urban areas within a city and in achieving the urban sustainability for now and for next generations, creating a more dynamic spatial urban experience $[6,7]$.

Scoping the sustainability index in historical urban areas [7], further depth is highlighted in the research literature review referring to the detailed approach of existing indexes, as follows. 


\subsubsection{BEQUEST framework}

Evaluations of the sustainable urban development indicators model BEQUEST sets out the various types of assessment methods that could easily be defined for essential characteristics [1].

Table 1: Bequest framework.

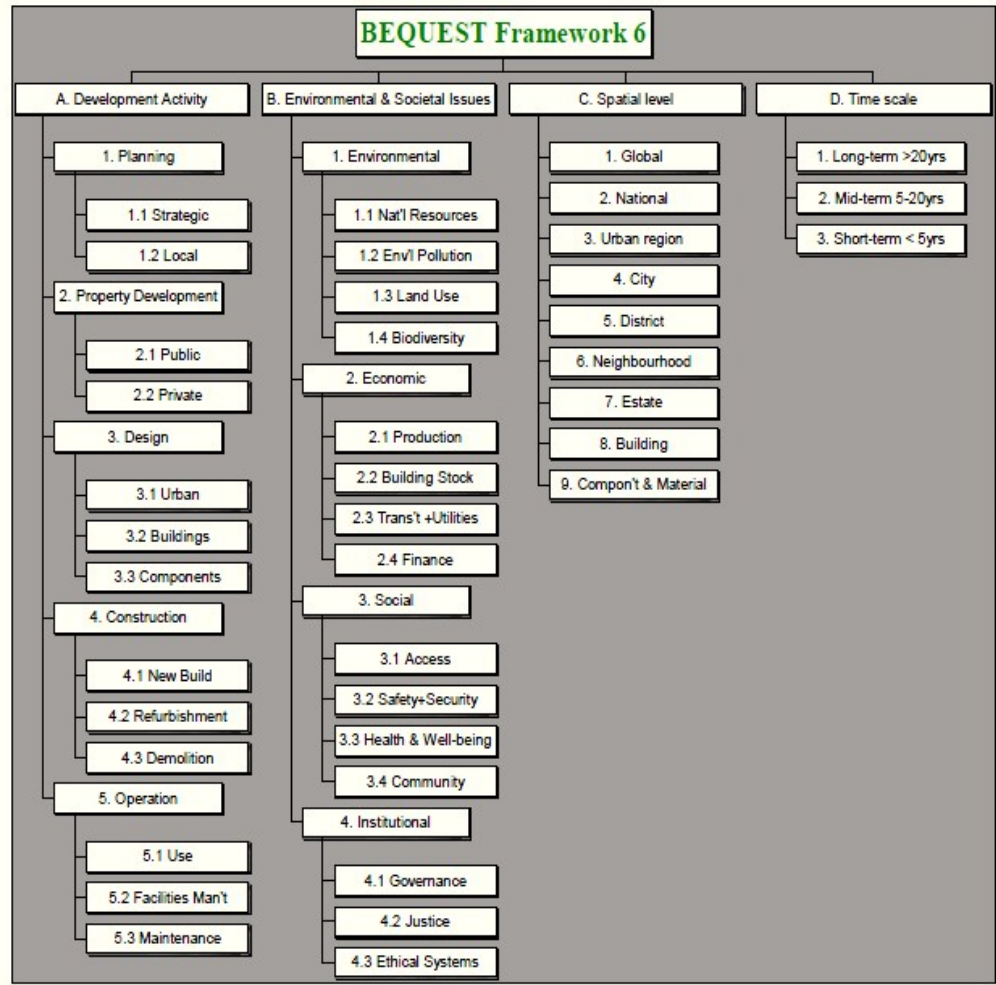

In this context, the BEQUEST framework is a 'vision' and 'methodology' of a collaborative platform described through a group of variables and supported by a set of protocols to use for assessments in a decision support system evaluating the sustainability of an urban setting [8]. The research will customize its approach towards historical urban areas by merging the quantifiable city approach and taking into consideration the psychological variables $[8,9]$.

The framework relates to four main dimensions of urban sustainability: the development activity, environmental and social issues, spatial level; and timescale, according to table 1 , and described as follows.

2.2.1.1 Development activity defined as a process This demonstrates good practice guidance for sustaining historical areas uses and functions compulsory with the urban sustainability initiative starting with strategic planning on the one 
hand to utilization of the resulting built environment on the other, which is essential to the approach of conservation and preservation [9].

The main activities and their sub-activities, i.e. planning (strategic and local, respecting the organic fabric), property development (public and private interests), design (urban, building and components), construction (new build, refurbishment and demolition, re-use of historical buildings), and operation (use, facilities management and maintenance, services and infra-structure), each of these aspects represent separate processes; affecting urban sustainability of historical areas trends, good practice and assessment has to be applied $[8,9]$.

2.2.1.2 Environmental and social issues Various human activities create effects with a relevant sustainability level. These activities produce subsidiary consequences of environmental, economic and social stresses. Environmental stresses include depletion of natural resources, pollution, and excessive land use which has negative effect on historical buildings as well as all the urban setting. Economic stress is often a cause of loss of production, decaying building stock, and/or of incentives [9]. Transport and utilities are important industrial sectors that affect and are affected by other economic sectors [10]. Social stress may include lack of access to facilities, inadequate safety and security, poor health or general loss of well-being which is often associated with poor sense of community. Good governance is necessary to create equality of access to resources along with social participation and judicial means of redress are all part of the institutional framework necessary to support urban sustainability in historical areas [10]. These cited aspects, in addition to the spiritual dimensions of life, form an ethical paradigm [11].

2.2.1.3 Spatial levels Urban sustainability of historical areas can take place at various spatial levels from the scale of the whole urban setting down to the individual historical building, its construction systems and material components [10]. Equally, the environmental effects or other socio-economic implications can be felt from local to global levels. A planning proposal can lead to various new industrial and commercial impacts on the environment, economy and society; this should shield from the level of the historical urban setting down to the neighborhood, and the historical building scale [10].

2.2.1.4 Timescale The importance of long-term planning regarding urban sustainability in historical areas has been emphasized through the prominence of the assessment approach [10]. The timescale parameters implemented by BEQUEST, i.e. short-term 0-5 years, medium-term 5-20 years and long-term more than 20 years, represent the normal scale used in economic and strategic planning, relevant to progressive stages of evaluation and re-assessment [10].

\subsubsection{Psychological aspects}

Psychological aspects investigate the individuals' contexts pertinent to the addressed community within specified historical urban areas. The main aim is to understand and enhance the quality of life through collaborative research and 
action plans, merging the psychological aspects with the urban variables of historical urban settings [10].

2.2.2.1 Sense of place This can be defined as the emotional and historical integrations resulting due to the existence of certain urban themes, provision of comfort and shade, relaxation, link to nature, topography and vegetation, added to this are the simple urban understanding, way finding and initial location contributing to spatial identification [10]. The sense of place is made up of a web consisting of a number of buildings and streets, from different historical periods, which creates a variety of cultural and urban strata. Over the centuries, these webs have defined the spatial urban character, and now offer a place that cannot be duplicated [8]; due to the circumstances that shaped and formed a unique and special place, giving it characteristics gained through political, geographical and socio-economic factors.

2.2.2.2 Locality and identity Locality and identity, which are defined as the feeling of belonging to a certain urban context, as part of the self-conception and self-perception of a local community within its urban settings. This feeling of belonging usually increases especially within historical areas; in which every local community seeks an expression of personalization, defined by different ways in colors, and materials, in addition to claiming their local territoriality, personal space, and bubble of privacy [8]. The self-evidence of a locality, in which one experiences a unique sense of place, remains important [10].

2.2.2.3 Cultural and spiritual aspects These represent the psychological values of local communities related to their urban setting. Culture can be seen as a part of the historical reservoir, which has great meaning regarding the local community. Spiritual values of historical urban areas refer to the significant features of history. This sense of touching a deeper realistic meaning increases the community vitality and motivates them to care and conserve their historical urban areas [10]. Cultural and spiritual concepts have a significance of inspiration, and symbolic interpretation of local identity. Therefore spiritual aspects are considered one of the major indicators of cultural identity, and the urban intellectual heritages forming framework for each society's unique understanding of life (UNESCO 1993) [10].

2.2.2.4 Aesthetic qualities These can be can be defined not only as the theory of beauty, but also as providing luxury towards human needs, feeling of comfort, safety and security [10]. In addition to that, the happiness gained from experiencing a beautiful place, sensing its joy, helping an easy way to find urban cognition and perceptions. These are realized by applying adequate proportions, colors and materials, considering the human scale, providing human necessities, diligent means of safety and the feeling of protection and security [2].

The existence of human pleasure and comfort is considered to be an urban quality within a non-physical approach which helps in analyzing human requirements and fulfilling their needs in order to provide daily life standards 
within the surrounding environment [10]. Since beauty is in the eye of the beholder, a well-designed urban settlement must be to address user-defined beauty. Investigating the human needs is the only way to realize their demands for living and enhance their perception of beauty; therefore aesthetic qualities are a universal human need to experience beauty.

\section{Sustainable historical area index (SHAI)}

The research proposed model SHAI is an index based on the BEQUEST framework and the quantifiable city theories, described in points 1 and 2 above, in addition to the local community psychological aspects, and taking into consideration the decision making process within key factors representing government institutions and local communities and non-governmental organization 'NGOs' [11].

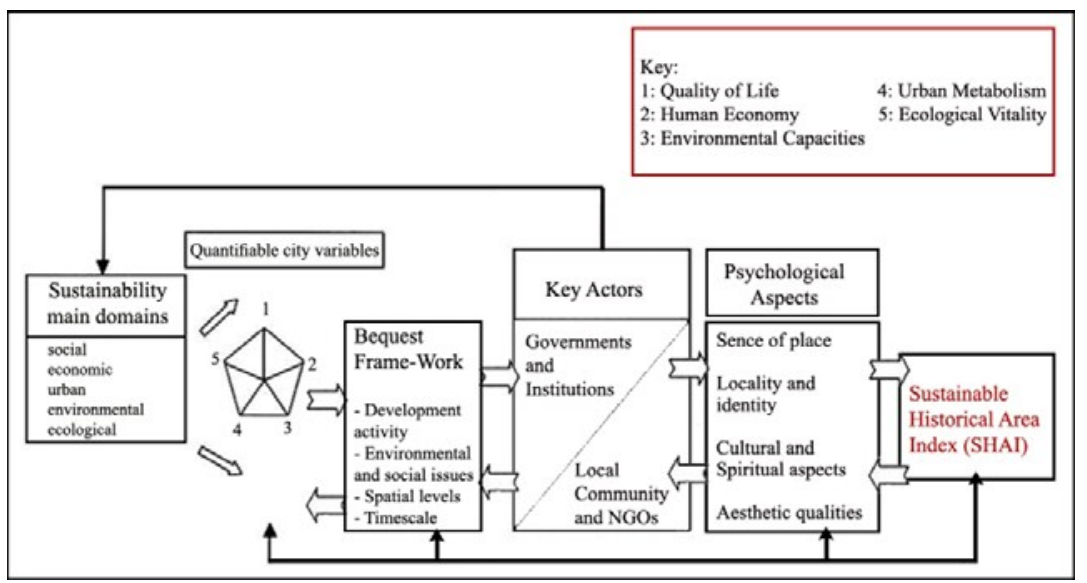

Figure 3: Sustainable historical area index (SHAI).

The model represents SHAI modeling stages, starting with Stage One, defining the urban sustainability main domains (social, economic, ecology, urban and environmental). Stage Two, and Stage Three, where the model merges two theories as based on the literature review to obtain the first nine variables of the sustainability historical area index (SHAI); (human economy, urban metabolism, ecological vitalities, quality of life, environmental capacities, development activities, environmental and social issues, spatial levels, and time-scale) [10].

Stage Four is considered 'as the intermediate stage of filtration' refers to the key-actors role, both the government institutions, and the local community in historical urban areas. This stage re-oriented the research towards the psychological dimension.

Stage Five added four more variables are added (sense of place, locality and identity, cultural and spiritual aspects, and aesthetic qualities).

The final stage, correlates all thirteen variables composing the sustainability historical area index (SHAI). 


\section{Methodological framework}

Figure 4 represents the seven steps of urban sustainability features analysis in historical settings. The methodology framework implemented within the SHAI Model is concluded from three main pillars: bequest, quantifiable city model and psychological aspects.

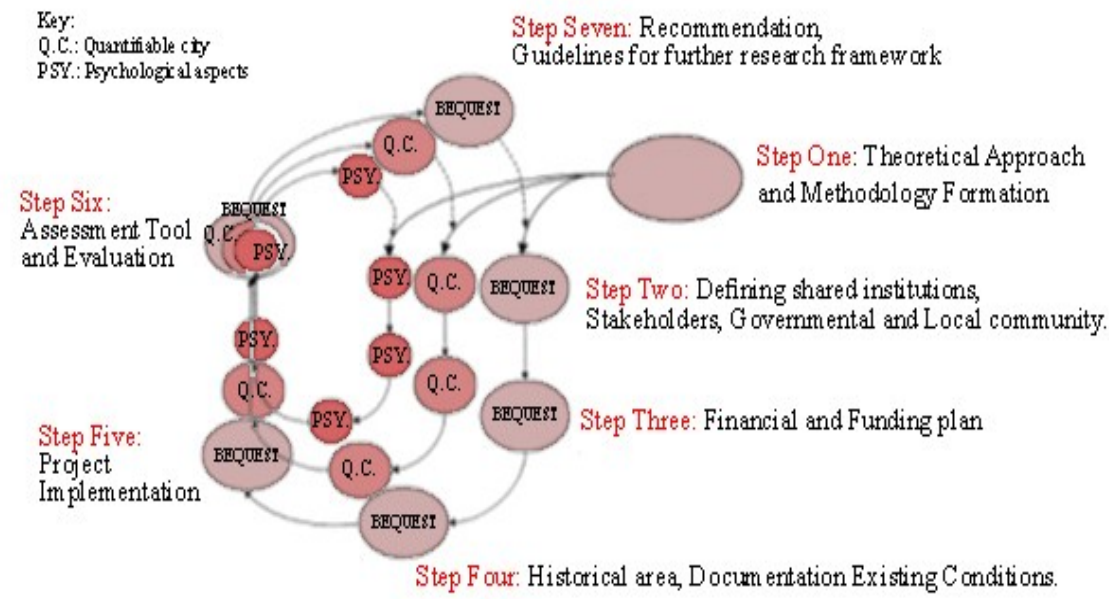

Figure 4: Urban sustainability framework in historical areas.

The methodological framework depends on seven steps: Step One represents the elementary phase of the theoretical approach formation, Step Two; determining stakeholders. Step Three; defining the funding plan. Step Four; urban documentation. Step Five; project implementation. Step Six; evaluation phase. Finally Step Seven; represents the resulting recommendations, and then sequentially closing the loop of the decision making process [11].

\section{Shai model for assessment methodology}

The sustainability historical areas index (SHAI) is presented through thirteen variables, concluded in table 2. These parameters are being evaluated based on statistical methodology according to their relative weights, which can be easily implemented on a specified case study approach. The experiment should be applied with relevant sampling techniques and data gathering based on observation, and semi-structure interviews [10]. Appropriate values are expected to be assigned to each variable $(\mathrm{X} 1, \mathrm{X} 2 \ldots \mathrm{Xn})$ and gives a value to each domain of the (SHAI) $=$ YN, (social, urban... etc.,) Scale as: Inconvenient: 0-5\%, Moderate: 6-10\%, Good: 11-15\%, Convenient: 16-20\%, [12]. 
Table 2: Assessment methodology applied on SHAI.

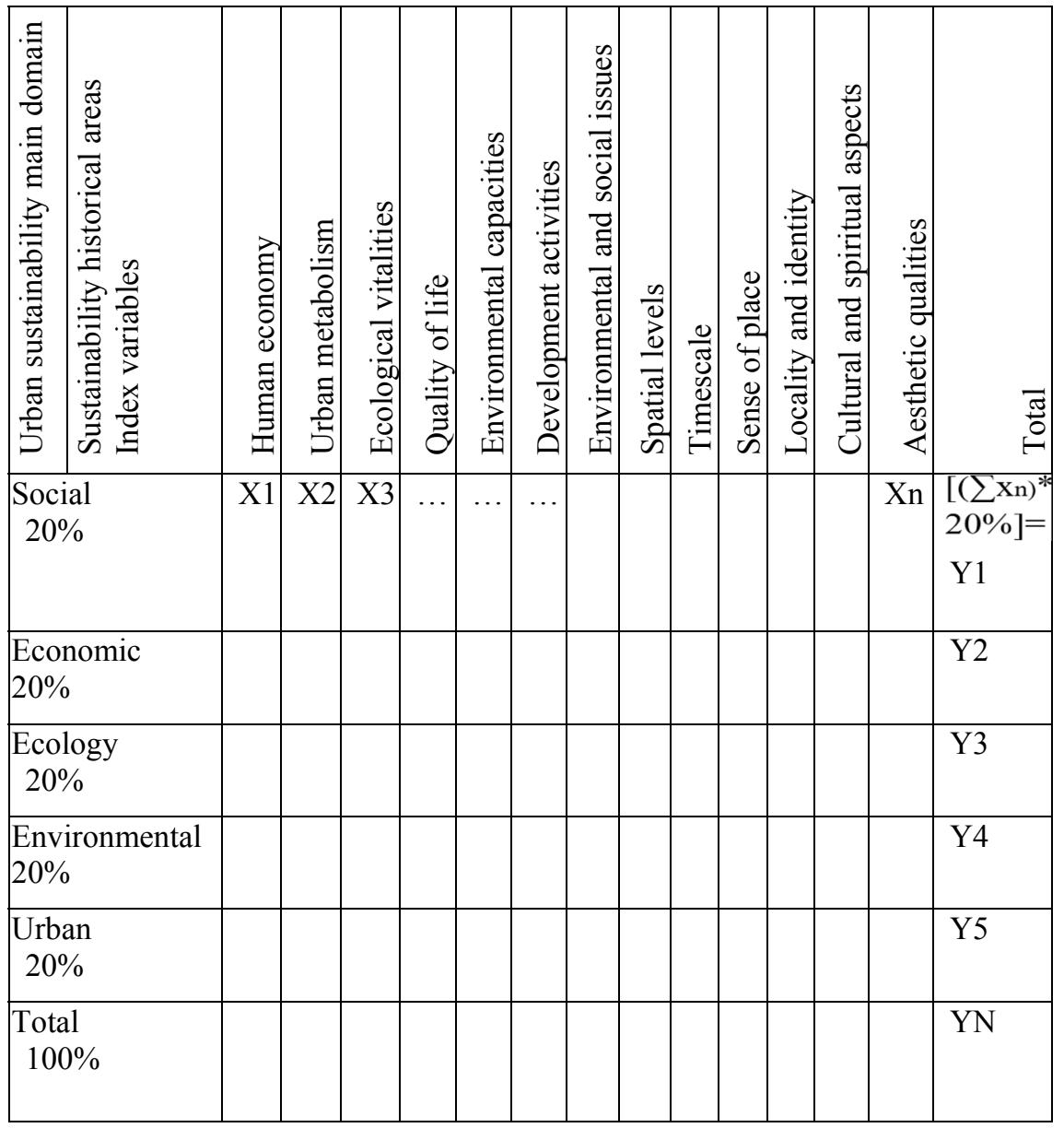

\section{Conclusion}

The optimization of urban sustainability in historical areas is determined by indispensable approaches towards establishing communities more oriented towards fulfilling the users' needs and requirements. It must take into consideration conserving the historical urban setting, in terms of building, urban fabric, and local residents' activities.

The developed model of "SHAI" - sustainable historical areas index, is considered as a wide-ranging set of indicators for historical urban sustainability features. Therefore, the model ensures the idea that a city can offer quality of life based on assessed variables implying a number of sustainability principles. This comprehensive vision merges both tangible and intangible dimensions, of the physical and non-physical urban qualities, in conserved historical areas. 
The methodology adopted to present a set of guidelines assessing the impact of urban development and procedures can be implemented in testing areas should consider:

a. "screening" urban use and social activities; the existing situation;

b. "scoping" key urban sustainable assets;

c. "clarifying" the types of activities, environmental, economic and social issues that need to be addressed;

d. carrying out the required "consultations" with interested parties;

e. "procuring" environmental assessments of urban sustainability plans, programs and projects.

The research will further contribute to "assessing" the urban sustainability variables in historical settings, plans, programs and projects and build the capacity which cities need to carry their cultural heritage and produce sustainable forms of human settlement.

The model also allows for professional knowledge and deepening academic understanding by monitoring the process of urban sustainability involvement as well as considering the stakeholder beneficiary preferences.

Therefore, SHAI, the multi scalar indicators delivered scheme incentives is actually adopting assessment methods. The model implementation will achieve more effective developments in sustaining historical areas and providing such quantifiable assessment enhancing the development of urban, social, environmental, ecological, and economical aspects.

\section{References}

[1] Vincenzo B., et al. A vision and methodology for integrated sustainable urban development: BEQUEST, Building Research and Information, 2002. http://dx.doi.org/10.1080/096132102753436468

[2] Mark D. et al. Sustainable Cities and Sustainable urban development: Use of the environmental assessment methods, Journal of Sustainable Cities and Society, vol. 10, pp. 39-48, Elsevier, 2014. www.elsevier.com/locate $/ \mathrm{scs}$

[3] Florianna L. et al. Review of urban sustainability indicators assessment $\mathrm{e}$ Case study between Asian countries, Habitat International vol. 44, pp. 491500, 2014. www.elsevier.com/locate/habitatint 2014

[4] Rahul B., Indicator-based urban sustainability-A review, Energy for Sustainable Development vol.17, pp: 555-563, International Energy Initiative, Elsevier, 2013. http://dx.doi.org/10.1016/j.esd.2013.08.004

[5] López-Ridaura S., et al. Multiscale Methodological Framework to Derive Criteria and Indicators for Sustainability Evaluation of Peasant Natural Resource Management Systems, Environment, Development and Sustainability, vol. 7, pp. 51-69, Springer, 2005. DOI 10.1007/s10668003-6976-x.

[6] United Nations Development Program. www.undp.org 
[7] Guilherme C. et al. Defining best practices in Sustainable Urban Regeneration Projects, Building Sustainability Assessment Tools, Portugal SB13 - Contribution of Sustainable Building to Meet EU 20-20-20 Targets, 2013.

[8] An Indicator-based Approach to Measuring Sustainable Urban Regeneration Performance: Part 1, Conceptual Foundations and Methodological Framework, Urban Studies, Vol. 41, No. 4, pp. 725-755, April 2004.

[9] Lesley H., An Indicator-based Approach to Measuring Sustainable Urban Regeneration Performance: Part 2, Empirical Evaluation and Case-study Analysis, Urban Studies, Vol. 41, No. 4, pp. 757-772, April 2004.

[10] Abdelfattah, D. Quantitative analysis for urban qualities in conserved areas, (Unpublished master's thesis). Cairo University, Egypt, 2011.

[11] A. Nayer et al. Building Regulation tester application, Beirut University Journal, 2009.

[12] A. Nayer et al. Urban Fabric formulation and the building design, Beirut University Journal, 2009. 